\title{
ROBUST MULTIVARIABLE SET-POINT REGULATION VIA STABLE DYNAMIC INVERSION
}

\author{
Aurelio Piazzi ${ }^{*}$ Antonio Visioli ${ }^{* *}$ \\ * Dipartimento di Ingegneria dell'Informazione, University of Parma \\ (Italy),e-mail: aurelio@ce.unipr.it \\ ** Dipartimento di Elettronica per l'Automazione, University of \\ Brescia, (Italy),e-mail:visioli@bsing.ing.unibs.it
}

\begin{abstract}
In this paper we propose a design methodology for the robust regulation of uncertain nonminimum-phase multivariable linear systems. The method basically consists of defining a suitable vector of parameterized desired output functions and of designing a parameterized decoupling controller of the nominal plant. Then, a parameterized command function for each input is determined by means of a stable dynamic inversion procedure. Eventually, solving an optimization problem permits to minimize the worst-case settling time, subject to amplitude limits on the control variables and constraints on the overshoots and undershoots of the outputs. A worked example based on a three-inputs three-outputs plant shows the effectiveness of the methodology. Copyright ${ }^{(\mathcal{C}} 2002$ IFAC
\end{abstract}

Keywords: Multivariable set-point regulation, dynamic inversion, constrained control, structured uncertainties, optimization.

\section{INTRODUCTION}

Despite the robust regulation of linear systems affected by parametric uncertainties has received much attention in the research community, the determination of design procedures for the synthesis of controllers which are able to achieve predefined performances in regulating uncertain systems is a problem which has not been solved satisfactorily yet. In this context, we consider a multi-input-multi-output (MIMO) nonminimum-phase system subject to structured uncertainties and we propose a new design tool for the synthesis of a feedforward/feedback control scheme for its robust set-point regulation. In particular, we search for a feedback controller and a vector of command functions in order to minimize the worst-case settling time subject to arbitrarily given overshoot and undershoot constraints and to amplitude limits for the plant control variables.

The devised solution is based on the concept of dynamic inversion (see e.g. (Hunt et al., 1996)) and significantly extends the method we proposed in other papers for the single-input-single-output (SISO) case
(Piazzi and Visioli, 2001c; Piazzi and Visioli, 2001a). Basically, the method consists of first defining for each output a sufficiently smooth desired output function (the "transition polynomials" investigated in (Piazzi and Visioli, 2001b)) which depends on the transition time $\tau$. Then, a parameterized feedback controller is determined with the aim of both decoupling the nominal plant and ensuring the internal model principle in order to achieve a zero steady-state error. At this point, a parameterized input command function can be calculated for each nominal decoupled SISO system by means of a stable inversion procedure. Finally, solving an optimization problem permits to find the design parameters that minimize the worst-case settling time subject to the selected constraints.

The general main idea behind the presented methodology is the combined synthesis of the feedback controller and of the command signals. This differs from the classical use of dynamic inversion for output tracking, where the dynamic inversion is performed on the nominal plant and the feedback controller, which aims at compensating initial condition mismatches, mod- 
eling errors and disturbances, is designed independently (Devasia, 2000). In our approach the dynamic inversion is applied to the SISO closed-loop systems and the main purpose of the feedback controller is to provide a good decoupling and to reduce the effects of the uncertain parameters on the closed-loop transfer functions.

\section{THE MULTIVARIABLE SET-POINT CONSTRAINED REGULATION PROBLEM}

We consider a linear, time-invariant, continuous-time uncertain MIMO system whose matrix transfer function is:

$$
P(s ; \mathbf{q})=\left[P_{i j}(s ; \mathbf{q})\right] i=1, \ldots, m j=1, \ldots, m
$$

where $P_{i j}(s ; \mathbf{q})=b_{i j}(s ; \mathbf{q}) / a_{i j}(s ; \mathbf{q})$ are strictly proper, rational transfer functions nonlinearly depending on an uncertain parameter vector $\mathbf{q}=\left[q_{1}, \ldots, q_{l}\right]^{T}$ which belongs to a given multidimensional interval (box) $Q=\left[q_{1}^{-}, q_{1}^{+}\right]\left\{\quad \cdots\left\{\begin{array}{ll}\ddagger & \ddagger\end{array} q_{\delta}^{+}\right]\right.$where:

$$
\begin{aligned}
& a_{i j}(s ; \mathbf{q})=\sum_{k=0}^{h_{i j}} a_{i j, k}(\mathbf{q}) s^{i}, \\
& b_{i j}(s ; \mathbf{q})=\sum_{k=0}^{l_{i j}} b_{i j, k}(\mathbf{q}) s^{i}
\end{aligned}
$$

The order of $P_{i j}(s ; \mathbf{q})$ is $h_{i j}$, its relative order is $\rho_{i j}=$ $h_{i j}-l_{i j}$. Polynomials $a_{i j}(s ; \mathbf{q})$ are (Hurwitz) stable for all $\mathbf{q} \in Q$ and their coefficients are continuous nonlinear functions over $Q$. Moreover, the normal rank of $P(s ; \mathbf{q})$ is equal to $m$ for all $\mathbf{q} \in Q$ assuring in such a way the invertibility of the uncertain plant. Denote as $z_{P}(s ; \mathbf{q})$ the zero polynomial of $P(s ; \mathbf{q})$ and introduce the "nominal" parameter vector $\mathbf{q}^{0}:=\operatorname{mid}(Q)$, i.e. the midpoint center of the box $Q$. Then, in order to pose a sensible regulation problem we do the following assumptions.

Assumption 1. $z_{P}(0 ; \mathbf{q}) \neq 0 \quad \forall \mathbf{q} \in Q$.

Assumption 2. $z_{P}\left(s ; \mathbf{q}^{0}\right)$ does not have any imaginary roots.

The robust constrained regulation problem to be addressed can be posed as follows. Determine a feedforward/feedback control strategy in order to obtain for each system output $i$ a "robust" transition from a previous set-point value $y_{i}^{0}$ to a new one $y_{i}^{1}$. Without loss of generality in the following we will assume $y_{i}^{0}=0 \quad i=1, \ldots, m$. Obviously, the first requirement to be satisfied is the robust stability of the closed-loop over the uncertain domain $Q$. Moreover, both transitions have to satisfy an overshoot and an undershoot limitation, an amplitude constraint on the control variables $u_{i}(t)$ and the (worst-case) settling time has to be minimized.

In other words, the above problem can be stated as follows: determine $m$ command functions $\mathbf{r}(t)=$

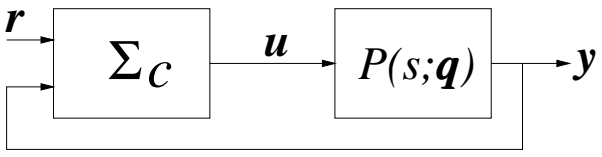

Fig. 1. The general control architecture.

$\left[r_{1}(t) \cdots r_{m}(t)\right]^{T}$ and a controller $\Sigma_{c}$ (see Figure 1) such that

(1) the closed-loop system is stable for all $\mathbf{q} \in Q$;

(2) $\lim _{t \rightarrow \infty} y_{i}(t)=y_{i}^{1}$ for $i=1, \ldots, m$ and for all $\mathbf{q} \in$ $Q$ (steady-state condition);

(3) the overshoot of the $i$ th output in response to $\mathbf{r}(t)$ is bounded by a given $\bar{O}_{i}$ for $i=1, \ldots, m$ and for all $\mathbf{q} \in Q$;

(4) the undershoot of the $i$ th output in response to $\mathbf{r}(t)$ is bounded by a given $\bar{U}_{i}$ for $i=1, \ldots, m$ and for all $\mathbf{q} \in Q$;

(5) the absolute value of the manipulative inputs $u_{i}(t)$ is bounded by a given $u_{i}^{s}$ for $i=1, \ldots, m$ and for all $\mathbf{q} \in Q$;

(6) it is minimized the worst-case settling time.

It appears that finding a global solution of the posed problem is a very hard task. In any case, we propose a design methodology, based on the concept of stable dynamic inversion, that provides a valuable suboptimal solution.

\section{A NEW INVERSION-BASED MULTIVARIABLE CONTROL ARCHITECTURE}

Figure 2 depicts the proposed control architecture to achieve an optimal set-point regulation under all the specifications given in the previous section. In the inner loop the controller $C_{1}(s)$ is designed to obtain a complete nominal decoupling from variable $\mathbf{v}$ to output $\mathbf{y}$, i.e. the nominal transfer function from $\mathbf{v}$ to $\mathbf{y}$ is diagonal. Moreover, $C_{1}(s)$ has to guarantee robust stability of the closed-loop from $\mathbf{v}$ to $\mathbf{y}$ for all $\mathbf{q} \in Q$. The controller $C_{2}(s)$ is the "regulating" unit ensuring the internal model principle to get steady-state robust regulation. It is synthesized according to

$$
C_{2}(s ; \boldsymbol{\alpha}):=\frac{1}{s} \operatorname{diag}(\boldsymbol{\alpha})
$$

where $\boldsymbol{\alpha}:=\left[\alpha_{1}, \ldots, \alpha_{m}\right]^{T} \in \mathbb{R}^{+m}$ is a vector gain to be eventually determined by solving the optimization problem of the next section. The devised outputs are chosen to be "transition" polynomials of (Piazzi and Visioli, 2001b). In the time interval $[0, \tau]$ they can be defined as follows $(i=1, \ldots, m)$ :

$$
\begin{aligned}
& y_{i}(t ; \tau):= \\
& y_{i}^{1} \frac{(2 p+1) !}{p ! \tau^{2 p+1}} \sum_{i=0}^{p} \frac{(-1)^{p-i}}{i !(p-i) !(2 p-i+1)} \tau^{i} t^{2 p-i+1}
\end{aligned}
$$

For $t<0$ it is $y_{i}(t ; \tau):=0$ and For $t>\tau$ it is $y_{i}(t ; \tau):=y_{i}^{1}$. These ideal output functions $y_{i}(t ; \tau)$ permit a smooth transfer from 0 to $y_{i}^{1}$ in the time interval $[0, \tau], \tau \in \mathbb{R}^{+}$without undershooting nor overshooting. Moreover $y_{i}(t ; \tau) \in C^{p}(\mathbb{R})$ with continuity order $p$ 


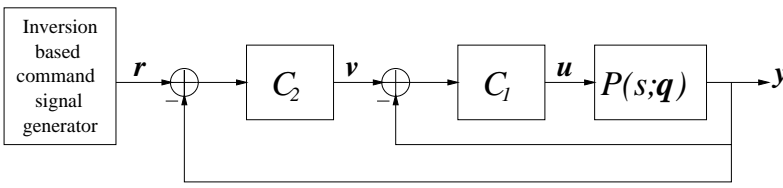

Fig. 2. The inversion-based multivariable control architecture.

being chosen according to a relative degree condition (see Proposition 4). The time interval parameter $\tau$ that greatly affects the actual settling time is eventually fixed by an optimization problem (16). The command signals $r_{i}$ are determined by performing a stable dynamic inversion from desired outputs $y_{i}(t ; \tau)$ over the nominal overall closed-loop system (see subsection 3.2). By virtue of the chosen structures for controllers $C_{1}$ and $C_{2}$ this results in a set of scalar inversions.

\subsection{The decoupling controller and robust stability properties}

The nominal zero polynomial $z_{p}\left(s ; \mathbf{q}^{0}\right)$ of plant $P\left(s ; \mathbf{q}^{0}\right)$ be factorized as $z_{p}^{-}(s) z_{p}^{+}(s)$ where $z_{p}^{-}(s)$ and $z_{p}^{+}(-s)$ are Hurwitz stable, i.e. $z_{p}^{+}(s)$ has as roots all the "unstable" multivariable zeros of $P\left(s ; \mathbf{q}^{0}\right)$. We choose as nominal transfer function from $\mathbf{v}$ to $\mathbf{y}$ the following diagonal matrix:

$$
G(s ; \omega):=\operatorname{diag}\left(k_{i} \frac{z_{p}^{+}(s)}{d(s ; \omega)} ; \quad i=1, \ldots, m\right)
$$

where the denominator $d(s ; \omega)$ is chosen to be a Butterworth polynomial with bandwidth frequency $\omega \in$ $\mathbb{R}^{+}$. Without loss of generality, because of the sensible pairing of inputs and outputs at the outset of the plant definition, we assume $P_{i i}\left(0 ; \mathbf{q}^{0}\right) \neq 0$ and consequently define $(i=1, \ldots, m)$

$$
k_{i}:=\frac{\mu_{i}}{z_{p}^{+}(0)}\left|P_{i i}\left(0 ; \mathbf{q}^{0}\right)\right|
$$

where $\mu_{i}$ are real positive constants to be fixed in the interval $(0,1]$. Consider the nominal transfer function of the plant $P\left(s ; \mathbf{q}^{0}\right)$. Its inverse can be written as

$$
P^{-1}\left(s ; \mathbf{q}^{0}\right):=\frac{B(s)}{z_{p}^{-}(s) z_{p}^{+}(s)}
$$

where $B(s)$ is a polynomial matrix. Define $\sigma_{i j}:=$ $\operatorname{deg}\left[B_{i j}(s)\right]-\operatorname{deg}\left[z_{p}\left(s ; \mathbf{q}^{0}\right)\right], i, j=1, \ldots, m$ and the total relative degree as $\sigma:=\max \left\{\sigma_{i j}: i, j=1, \ldots, m\right\}$. Then, we set the degree of the Butterworth polynomial $d(s ; \omega)$ according to:

$$
\operatorname{deg}[d(s ; \omega)]:=\operatorname{deg}\left[z_{p}^{+}(s)\right]+\sigma .
$$

Proposition 3. The decoupling controller $C_{1}$ be defined by

$$
C_{1}(s ; \omega):=P^{-1}\left(s ; \mathbf{q}^{0}\right)(I-G(s ; \omega))^{-1} G(s ; \omega) .
$$

Then, the nominal transfer function from $\mathbf{v}$ to $\mathbf{y}$ is exactly given by (5). Moreover, if all the unstable polezero cancellations are performed in (9) to determine $C_{1}$, then the nominal closed-loop system from $\mathbf{v}$ to $\mathbf{y}$ is internally stable.

Proof. Given a controller $C_{1}$, the transfer function from $\mathbf{v}$ to $\mathbf{y}$ is $\left(I+P C_{1}\right)^{-1} P C_{1}$. Then, by insertion of expression (9) we obtain

$\left[I+(I-G(s ; \omega))^{-1} G(s ; \omega)\right]^{-1}(I-G(s ; \omega))^{-1} G(s ; \omega)$.

By taking into account that

$$
I+(I-G(s ; \omega))^{-1} G(s ; \omega)=(I-G(s ; \omega))^{-1}
$$

we eventually recognize expression (10) as the desired diagonal matrix $G(s ; \omega)$. Now, consider the structure of controller $C_{1}$ as it results from (9), (5) and (7). First, note that

$$
\begin{aligned}
& (I-G(s ; \omega))^{-1} G(s ; \omega)= \\
& \quad \operatorname{diag}\left(\frac{k_{i} z_{p}^{+}(s)}{d(s ; \omega)-k_{i} z_{p}^{+}(s)} ; i=1, \ldots, m\right) .
\end{aligned}
$$

Then, performing all the unstable pole-zero cancellations in the expression (9), the controller $C_{1}(s ; \omega)$ emerges as $(i, j=1, \ldots, m)$ :

$$
C_{i j}(s ; \omega)=\frac{k_{j} B_{i j}(s)}{z_{p}^{-}(s)\left[d(s ; \omega)-k_{j} z_{p}^{+}(s)\right]} .
$$

From the above expression (13) we deduce that only allowable stable pole-zero cancellations are made between $C_{1}(s ; \omega)$ and the nominal plant $P\left(s ; \mathbf{q}^{0}\right)$. Hence, the internal stability of the nominal closed-loop system from $\mathbf{v}$ to $\mathbf{y}$ is ensured by the Hurwitz stability of the Butterworth polynomial $d(s ; \omega)$ characterizing the poles of $G(s ; \omega)$.

The next result enforces the internal stability of the closed-loop from $\mathbf{v}$ to $\mathbf{y}$ over the entire family of uncertain plants.

Proposition 4. There exists constants $\mu_{i} \in(0,1], i=$ $1, \ldots, m$ such that internal stability of the closed-loop from $\mathbf{v}$ to $\mathbf{y}$ holds for all $\mathbf{q} \in Q$.

Proof. By virtue of the definition of $k_{i}$ in (6) it is possible to choose $\mu_{i} \in(0,1]$ in order to make $\left|k_{i}\right|$ as small as desired. By taking into account the structure of the controller $C_{1}$ given in (13) it is apparent that the inauence of $C_{1}$ versus the internal stability of the closed-loop from $\mathbf{v}$ to $\mathbf{y}$ becomes negligible when all the $k_{i}$ 's turn to be arbitrarily small. Considering that $P(s ; \mathbf{q})$ is stable for all $\mathbf{q} \in Q$ it follows the statement of Proposition 4.

Building on the previous Proposition 4 we can state the following robustness result.

Proposition 5. There exist constant $\mu_{i} \in(0,1]$ and integrator gains $\alpha_{i}>0, i=1, \ldots, m$ such that internal stability of the overall closed-loop system from $\mathbf{r}$ to $\mathbf{y}$ holds for all $\mathbf{q} \in Q$. 
Proof. A sketch of proof is offered. First choose constants $\mu_{i} \in(0,1]$ according to Proposition 2 in order to get the robust internal stability of the closed-loop from $\mathbf{v}$ to $\mathbf{y}$. Then, taking into account the definition of $k_{i}(6)$ and the structures of controller $C_{1}$ and $C_{2}$ as reported in (13) and (3) we deduce, by a root locus argument, that the internal stability of the overall closed-loop is guaranteed $\forall \mathbf{q} \in Q$ provided that the positive gains $\alpha_{i}$ are sufficiently small.

Remark 1. In defining the decoupled dynamics from $\mathbf{v}$ to $\mathbf{y}$, see (5), we have chosen as a sensible and feasible choice, the Butterworth polynomial $d(s ; \omega)$ with the frequency $\omega$ to be optimally fixed by optimization problem (16). At the discretion of the control designer, other choices could be done as well. Indeed, the robustness results of Propositions 4 and 5 still hold by substituting $d(s ; \omega)$ with any Hurwitz polynomial $d(s)$.

\subsection{Command signal synthesis via stable inversion}

Due to the chosen structures for controllers $C_{1}$ and $C_{2}$, see (3) and (13), the nominal transfer function from command signal $\mathbf{r}$ to output $\mathbf{y}$ is the diagonal matrix

$$
T(s ; \boldsymbol{\alpha}, \omega)=\operatorname{diag}\left(T_{i i}\left(s ; \alpha_{i}, \omega\right) ; i=1, \ldots, m\right)
$$

where

$$
T_{i i}\left(s ; \alpha_{i}, \omega\right)=\frac{\alpha_{i} k_{i} z_{p}^{+}(s)}{s d(s ; \omega)+\alpha_{i} k_{i} z_{p}^{+}(s)} .
$$

We want to determine the command signal $r_{i}$ as the input that causes the output $y_{i}$ to be the desired ideal function $y_{i}(t ; \tau)$ defined in (4). This calls for a stable dynamic inversion to be performed on a nominal system (15) (note that the system is nonminimumphase and therefore a standard dynamic inversion procedure cannot be applied, as the resulting input function would be unbounded). The set of all cause/effect function pairs $\left(r_{i}(\cdot), y_{i}(\cdot)\right)$ associated to $T_{i i}\left(s ; \alpha_{i}, \omega\right)$ be denoted by $\mathcal{B}_{i}$. A relevant property of $\mathcal{B}_{i}$ is given by the following result (see (Piazzi and Visioli, 2001b)).

Proposition 6. Let us consider a pair $\left(r_{i}(\cdot), y_{i}(\cdot)\right) \in \mathcal{B}_{i}$. Then, $r_{i}(\cdot) \in C^{l}(\mathbb{R})$ if and only if $y_{i}(\cdot) \in C^{l+\sigma+1}(\mathbb{R})$ where $\sigma+1$ is the total relative degree of system (15) (see definition (8)).

Relying on the above proposition and taking into account that $y_{i}(\cdot) \in C^{p}(\mathbb{R})$ we set $p:=\sigma+1$ in order to synthesize a continuous command signal. Now, by following the methodology explained in (Piazzi and Visioli, 2001a), we are able to synthesize $i$ functions $r_{i}\left(t ; \tau, \alpha_{i}, \omega\right)$, defined over $(-\infty,+\infty)$ that exactly solve the stable dynamic inversion problem, i.e. that exactly causes the desired output $y_{i}(t ; \tau)$. In order to practically use the synthesized function $r_{i}\left(t ; \tau, \alpha_{i}, \omega\right)$ it is necessary to truncate it, resulting in an approximate generation of the desired output $y_{i}(t ; \tau)$. This can be done with arbitrarily precision (Piazzi and Visioli, 2001a). The approximated command functions be denoted by $r_{i}^{a}\left(t ; \tau, \alpha_{i}, \omega\right)(i=1, \ldots, m)$. Note that this stable dynamic inversion procedure can yield to the so-called "preaction control" (Marro, 1996).

\section{WORST-CASE DESIGN VIA SEMI-INFINITE OPTIMIZATION}

In the previous section we have synthesized all the structures of the inversion-based multivariable control architecture: the decoupling controller $C_{1}$ that depends on $\omega \in \mathbb{R}^{+}$and $\mu_{i} \in(0,1]$, see (9) and (6); the regulating unit $C_{2}$ with integrator gains $\alpha_{i}, i=1, \ldots, m$; and the command inputs $r_{i}^{a}\left(t ; \tau, \alpha_{i}, \omega\right), i=1, \ldots, m$ determined via stable dynamic inversion on a family of ideal output transfers $y_{i}(t ; \tau)$. The design procedure can be then organized as follows:

(1) Choose constants $\mu_{i} \in(0,1]$ in such a way robust stability of the internal closed-loop from $\mathbf{v}$ to $\mathbf{y}$ holds for all $\mathbf{q} \in Q$.

(2) Determine the optimal controller parameters $\omega^{*}$, and $\alpha_{1}^{*}, \ldots, \alpha_{m}^{*}$ and optimal output transition interval $\tau^{*}$ that minimize the worst-case settling time subject to all the required control and setpoint specifications.

The design phase 1 relies on Proposition 2 and on the practical side we can set all the $\mu_{i}$ 's equal to 1 and if robust stability does not hold we make the $\mu_{i}$ 's smaller and smaller until robustness is ensured. To this end, for checking the robust stability a variety of tools is available, for example (Balakrishnan et al., 1991; Bhattacharyya et al., 1995; Piazzi and Marro, 1996).

In the second and final design phase a semi-infinite optimization problem emerges without any conservativeness and an approximate solution can be obtained by using genetic algorithms. Robust stability of the overall closed-loop can be taken into account by means of the following result that can be derived, as shown in (Piazzi and Visioli, 2001a), from the Lienard and Chipard's criterion and Orlando's formula.

Proposition 7. The overall closed-loop system from $\mathbf{r}$ to $\mathbf{y}$ is stable for all $\mathbf{q} \in Q$ if and only if

$$
\begin{gathered}
S_{1}(\omega, \boldsymbol{\alpha}, \mathbf{q})>0, S_{2}(\omega, \boldsymbol{\alpha}, \mathbf{q})>0 \forall \mathbf{q} \in Q ; \\
S_{3}\left(\omega, \boldsymbol{\alpha}, \mathbf{q}^{0}\right)>0, \ldots, S_{n_{c}}\left(\omega, \boldsymbol{\alpha}, \mathbf{q}^{0}\right)>0 ;
\end{gathered}
$$

where $n_{c}$ is the degree of the characteristic pole polynomial of the overall closed-loop system and functions $S_{i}(\omega, \boldsymbol{\alpha}, \mathbf{q}), i=1, \ldots, n_{c}$ are associated coefficients or Hurwitz determinants as explained in (Piazzi and Visioli, 2001a).

Denote by $u_{i}^{a}(t ; \tau, \omega, \boldsymbol{\alpha}, \mathbf{q})$ and $y_{i}^{a}(t ; \tau, \omega, \boldsymbol{\alpha}, \mathbf{q}), i=$ $1, \ldots, m$ respectively the inputs and outputs of the plant when the command signals are $r_{i}^{a}\left(t ; \tau, \omega, \alpha_{i}, \mathbf{q}\right)$, $i=1, \ldots, m$. By virtue of the dynamic inversion approach adopted to synthesized the command signals we have that $y_{i}^{a}\left(t ; \tau, \omega, \boldsymbol{\alpha}, \mathbf{q}^{0}\right)$ is almost equal to $y_{i}(t ; \tau)$ 
(see the final sentences of subsection 3.2). Considering the presence of the regulating unit $C_{2}(s ; \boldsymbol{\alpha})$ ensuring the internal model principle, the steady-state requirement is always satisfied provided that the robust stability of the overall closed-loop is satisfied:

$$
\lim _{t \rightarrow \infty} y_{i}^{a}(t ; \tau, \omega, \boldsymbol{\alpha}, \mathbf{q})=y_{i}^{1} \forall \tau>0 \text { and } \forall \mathbf{q} \in Q .
$$

For the $i$ th output, the settling time $t_{i}^{s}(\tau, \omega, \boldsymbol{\alpha}, \mathbf{q})$ be defined as the minimum time (including the preaction time $\left|t_{0}\right|$ ) after that the regulated scalar output remains within a $2 \%$ range of the desired steady-state value:

$$
\begin{aligned}
& t_{i}^{s}(\tau, \omega, \boldsymbol{\alpha}, \mathbf{q}):=\left|t_{0}\right|+ \\
& \min \left\{s \in \mathbb{R}^{+}:\left|y_{i}^{a}(t ; \tau, \omega, \boldsymbol{\alpha}, \mathbf{q})-y_{i}^{1}\right| \leq 0.02 y_{i}^{1} \quad \forall t \geq s\right\} .
\end{aligned}
$$

Taking a worst-case viewpoint, the (worst-case) settling time of the overall multivariable set-point transfer can be defined as:

$$
t_{w c s}(\tau, \omega, \boldsymbol{\alpha}) \max _{i=1, \ldots, m} \max _{\mathbf{q} \in Q}:=\left\{t_{i}^{s}(\tau, \omega, \boldsymbol{\alpha}, \mathbf{q})\right\} .
$$

Finding the optimal design parameters $\omega^{*}, \boldsymbol{\alpha}^{*}$, and $\tau^{*}$ entails solving the following semi-infinite optimization problem:

$$
\min \left\{t_{w c s}(\tau, \omega, \boldsymbol{\alpha}): \tau, \omega \in \mathbb{R}^{+}, \boldsymbol{\alpha} \in \mathbb{R}^{+m}\right\}
$$

subject to $(i=1, \ldots, m)$ :

$$
\begin{gathered}
S_{1}(\omega, \boldsymbol{\alpha}, \mathbf{q}) \geq \varepsilon, S_{2}(\omega, \boldsymbol{\alpha}, \mathbf{q}) \geq \varepsilon \quad \forall \mathbf{q} \in Q ; \\
S_{3}\left(\omega, \boldsymbol{\alpha}, \mathbf{q}^{0}\right) \geq \varepsilon, \ldots, S_{n_{c}}\left(\omega, \boldsymbol{\alpha}, \mathbf{q}^{0}\right) \geq \varepsilon ; \\
y_{i}^{a}(t ; \tau, \omega, \boldsymbol{\alpha}, \mathbf{q}) \leq\left(1+0.01 \bar{O}_{i}\right) y_{i}^{1} \forall t \geq 0 \quad \forall \mathbf{q} \in Q ; \\
y_{i}^{a}(t ; \tau, \omega, \boldsymbol{\alpha}, \mathbf{q}) \geq-0.01 \bar{U}_{i} y_{i}^{1} \forall t \geq 0 \quad \forall \mathbf{q} \in Q ; \\
\left|u_{i}^{a}(t ; \tau, \omega, \boldsymbol{\alpha}, \mathbf{q})\right| \leq u_{i}^{s} \forall t \geq 0 \quad \forall \mathbf{q} \in Q ;
\end{gathered}
$$

where the threshold constant $\varepsilon$ is a sufficiently small positive value. In solving (16) we can rely on the following result:

Proposition 8. For any given values of the overshooting and undershooting bounds $\bar{O}_{i}>0$ and $\bar{U}_{i}>0$, $i=1, \ldots, m$, the semi-infinite optimization problem (16) admits a solution if, for $i=1, \ldots, m$,

$$
\max _{\mathbf{q} \in Q}\left\{\text { ith component of } P^{-1}(0 ; \mathbf{q}) \mathbf{y}^{1}\right\}<u_{i}^{s}
$$

where $\mathbf{y}^{1}:=\left[y_{1}^{1}, \ldots, y_{m}^{1}\right]^{T}$.

Proof. Omitted for brevity. It is an extension of a proof appeared in (Piazzi and Visioli, 2001c).

Two remarks are in order on the above proposition. First, the condition (17) is well posed because $\operatorname{det} P(0 ; \mathbf{q}) \neq 0 \quad \forall \mathbf{q} \in Q$ by virtue of Assumption 1. Secondly, Proposition 8 comes as a sufficient condition but it can be also considered an almost necessary one. Indeed, if there exist an uncertain parameter $\overline{\mathbf{q}} \in Q$ and an index $\bar{i}$ for which $\left\{\right.$ the ith component of $\left.P^{-1}(0 ; \overline{\mathbf{q}}) \mathbf{y}^{1}\right\}>u_{\bar{i}}^{s}$ then the setpoint regulation problem is intrinsically not solvable regardless of the adopted control method. When this is the case it means that the plant static gain is too small to sustain in the steady-state the desired $\mathbf{y}_{\bar{i}}^{1}$.
Problem (16) is a difficult nonlinear semi-infinite optimization for which an approximate solution can be obtained by relaxing or discretizing the semi-infinite constraints. A simple way to do this is to substitute the box $Q$ with its vertexes and to use a genetic algorithm to finding estimates of $\omega^{*}, \boldsymbol{\alpha}^{*}$, and $\tau^{*}$ (Houck et al., 1995). This approach requires, as explained in (Piazzi and Visioli, 2001c), an algorithmic postprocessing to ensure the feasibility of the obtained approximate solution. A more effective but effortful approach is to adopt the genetic/interval algorithm of Guarino Lo Bianco and Piazzi (Guarino Lo Bianco and Piazzi, 2001) that provides estimates of the global minimizers and feasibility is guaranteed without any need of a post-processing validation phase.

\section{A DESIGN EXAMPLE}

As an illustrative example, consider the uncertain system $(m=3)$

$$
P(s ; \mathbf{q})=\left[\begin{array}{ccc}
\frac{2}{s+q_{1}} & \frac{1}{s+5} & 0 \\
0 & 2 \frac{s-q_{3}}{\left(s+q_{1}\right)\left(s+q_{2}\right)} & \frac{1}{s+3} \\
\frac{1}{s+q_{2}} & 0 & \frac{2}{s+q_{2}}
\end{array}\right]
$$

where $q_{1} \in[0.8,1.2], q_{2} \in[1.6,2.4], q_{3} \in[0.8,1.2]$. The resulting zero polynomial of $P\left(s ; \mathbf{q}^{0}\right)$ is:

$$
z_{p}\left(s ; \mathbf{q}^{0}\right)=s^{3}+\frac{60}{9} s^{2}+\frac{61}{9} s-\frac{118}{9}
$$

that means that there is a single right half plane zero in $z_{1}=0.941$. The total relative degree $\sigma$ is equal to one and therefore we selected a second order Butterworth polynomial:

$$
d(s ; \omega)=\frac{s^{2}}{\omega^{2}}+\sqrt{2} \frac{s}{\omega}+1 ;
$$

Then, we fixed $\mu_{i}=1, i=1,2,3$, and consequently we obtained $k_{1}=-0.152$ and $k_{2}=k_{3}=-0.076$, verifying that for those values the internal stability of the closed-loop from $\mathbf{v}$ to $\mathbf{y}$ holds (see Proposition 4). Having fixed the structure of the decoupling controller and of the regulating unit, we can perform the dynamic inversion procedure on the resulting three scalar systems (15) whose transfer functions depend on $\alpha_{i}, i=1,2,3$ and $\omega$. As desired output function, for all the three scalar outputs we adopted the transition polynomial of fifth order:

$$
y(t ; \tau)=\frac{6}{\tau^{5}} t^{5}-\frac{15}{\tau^{4}} t^{4}+\frac{10}{\tau^{3}} t^{3}, \quad t \in[0, \tau] .
$$

The optimization problem has been solved by means of genetic algorithms (Houck et al., 1995). We fixed $u_{i}^{s}=1.5, i=1,2,3$ and a maximum overshoot of $10 \%$ and a maximum undershoot of $5 \%$ for all the outputs. It results $\alpha_{1}^{*}=2.766, \alpha_{2}^{*}=5.277, \alpha_{3}^{*}=6.090, \omega^{*}=$ 


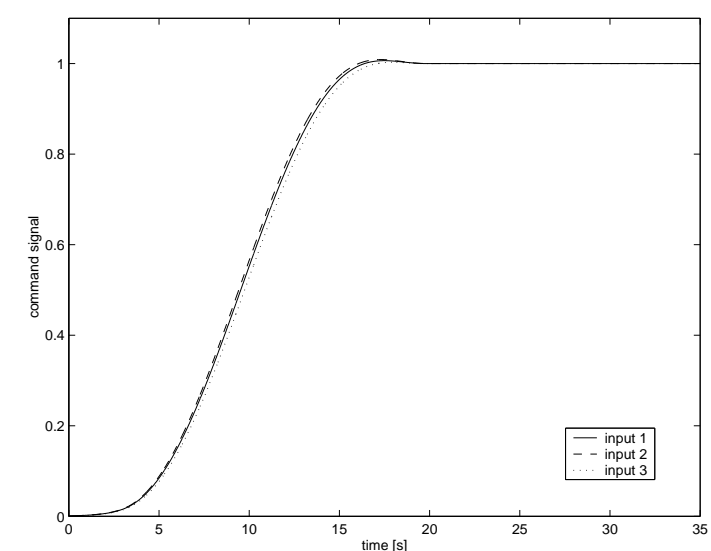

Fig. 3. The optimal command signals $r_{i}^{a}\left(t ; \tau^{*}, \alpha_{i}^{*}, \omega^{*}\right)$, $i=1,2,3$.

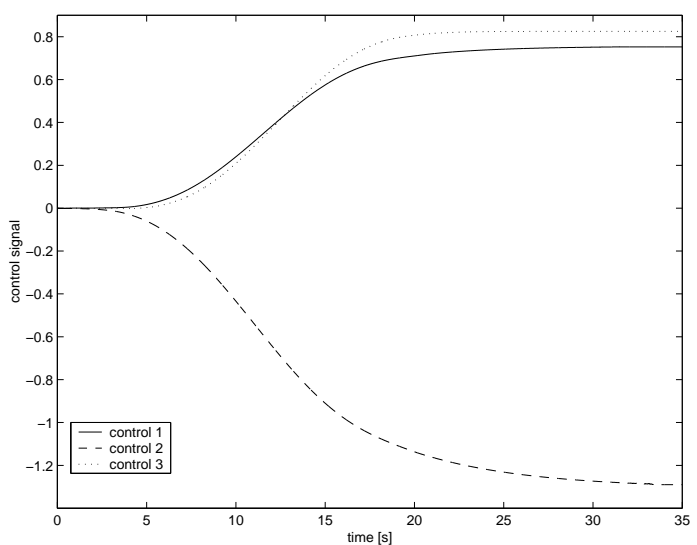

Fig. 4. The worst-case optimal control signals.

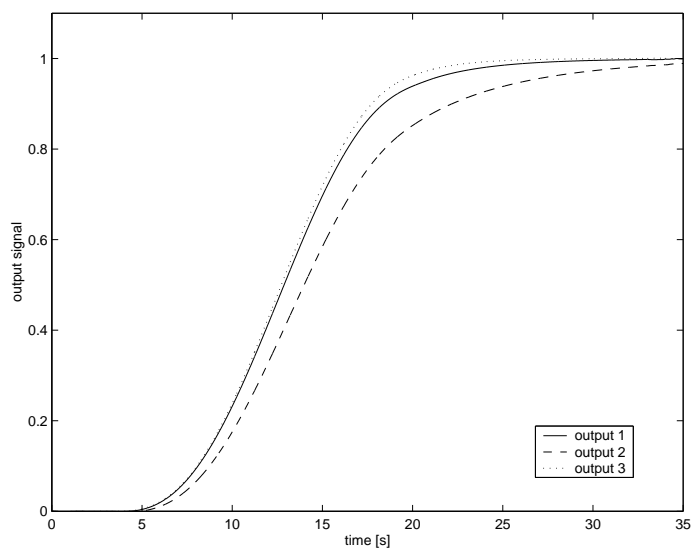

Fig. 5. The worst-case optimal output signals.

9.652 and $\tau^{*}=16.84$ and the corresponding worstcase settling time is $23.82 \mathrm{~s}$. The optimal command signals are plotted in Figure 3 and the corresponding worst-case control signals and system outputs are shown in Figures 4 and 5 respectively.

\section{CONCLUSIONS}

In this paper we have presented a design methodology, based on dynamic inversion, for the robust regulation of uncertain multivariable systems. The combined synthesis of the controller and of the command input functions, which is accomplished mainly by solving the optimization problem (16) is a strong point of the overall technique, because, basically, the feedback controllers significantly reduces the effects of the uncertainties and of the coupling and therefore permits to the stable dynamic inversion based command signal to improve the performances of the set-point transfers.

\section{REFERENCES}

Balakrishnan, V., S. Boyd and S. Balemi (1991). Branch and bound algorithm for computing the minimum stability degree of parameterdependent linear systems. Int. J. of Robust and Nonlinear Control 1, 295-317.

Bhattacharyya, S. P., H. Chapellat and L. H. Keel (1995). Robust control: the parametric approach. Prentice Hall.

Devasia, S. (2000). Robust inversion-based feedforward controllers for output tracking under plant uncertainty. In: Proceedings of the American Control Conference. Chicago, Illinois. pp. 497502.

Guarino Lo Bianco, C. and A. Piazzi (2001). A hybrid algorithm for infinitely constrained optimization. International Journal of Systems Science 32(1), 91-102.

Houck, C., J. Joines and M. Kay (1995). A genetic algorithm for function optimization: a matlab implementation. Technical Report NCSU-IE TR 95-09.

Hunt, L.R., G. Meyer and R. Su (1996). Noncausal inverses for linear systems. IEEE Transaction on Automatic Control AC-41, 608-611.

Marro, G. (1996). Multivariable regulation in geometric terms: old and new results. In: Colloquium on Automatic Control (C. Bonivento, G. Marro and R. Zanasi, Eds.). pp. 77-138. Springer.

Piazzi, A. and A. Visioli (2001a). Optimal inversionbased control for the set-point regulation of nonminimum-phase uncertain scalar systems. IEEE Trans. on Automatic Control 46(10), 16541659.

Piazzi, A. and A. Visioli (2001b). Optimal noncausal set-point regulation of scalar systems. Automatica 37(1), 121-127.

Piazzi, A. and A. Visioli (2001c). Robust set-point constrained regulation via dynamic inversion. International Journal of Robust and Nonlinear Control 11(1), 1-22.

Piazzi, A. and G. Marro (1996). Robust stability using interval analysis. International Journal of Systems Science 27(12), 1381-1390. 